\title{
Experimental study of proton emission from 60-fs, 200-mJ high-repetition-rate tabletop-laser pulses interacting with solid targets
}

\author{
I. Spencer, ${ }^{1}$ K. W. D. Ledingham, ${ }^{1,2, *}$ P. McKenna, ${ }^{1}$ T. McCanny, ${ }^{1}$ R. P. Singhal,,${ }^{1}$ P. S. Foster, ${ }^{3}$ D. Neely,${ }^{3}$ A. J. Langley,${ }^{3}$ \\ E. J. Divall, ${ }^{3}$ C. J. Hooker, ${ }^{3}$ R. J. Clarke, ${ }^{3}$ P. A. Norreys, ${ }^{3}$ E. L. Clark, ${ }^{2}$ K. Krushelnick, ${ }^{4}$ and J. R. Davies ${ }^{5}$ \\ ${ }^{1}$ Department of Physics and Astronomy, University of Glasgow, Glasgow G12 8QQ, United Kingdom \\ ${ }^{2} A W E$ plc, Aldermaston, Reading RG7 4PR, United Kingdom \\ ${ }^{3}$ Central Laser Facility, Rutherford Appleton Laboratory, Chilton, Oxfordshire OX11 OQX, United Kingdom \\ ${ }^{4}$ Blackett Laboratory, Imperial College, Prince Consort Road, London SW7 2BZ, United Kingdom \\ ${ }^{5}$ GoLP, Instituto Superior Técnico, 1049-001 Lisboa, Portugal
}

(Received 16 August 2002; revised manuscript received 26 November 2002; published 24 April 2003)

\begin{abstract}
Measurements of proton emission have been made from a variety of solid targets irradiated by a 60-fs, $200-\mathrm{mJ}, 7 \times 10^{18}-\mathrm{W} \mathrm{cm}^{-2}$ laser system operating at $2 \mathrm{~Hz}$. Optimum target conditions were found in terms of target material and thickness. For Mylar targets of thickness 20-40 $\mu \mathrm{m}$, a maximum proton energy of $1.5 \mathrm{MeV}$ was measured. For aluminum targets, a maximum energy of $950 \mathrm{keV}$ was measured for $12 \mu \mathrm{m}$, and for copper, $850 \mathrm{keV}$ for $12.5 \mu \mathrm{m}$.
\end{abstract}

DOI: 10.1103/PhysRevE.67.046402

PACS number(s): 52.38.Kd, 52.50.Jm

\section{INTRODUCTION}

Advances in laser technology such as chirped pulse amplification (CPA) [1,2] have led to the construction of multiterawatt and recently petawatt laser systems, and proposed techniques such as optical parametric chirped pulse amplification [3] promise to continue to extend these boundaries for the foreseeable future. Exciting new fields of research have developed in laser-matter interactions as a result of this technology. At intensities above about $10^{18} \mathrm{~W} \mathrm{~cm}^{-2}$ relativistic plasmas are created as electrons quiver in the laser field with energies equivalent to their rest mass. Beams of electrons and protons are accelerated and sources of neutrons, high fluxes of $\mathrm{x}$ rays, and high-energy $\gamma$ rays are created. Notable achievements including the laser-induced fission of ${ }^{238} \mathrm{U}$ $[4,5]$, the production of nuclear isotopes $[6,7]$, and the measurement of the highest magnetic fields ever produced in a laboratory [8] have ensured that this continues to be an exciting area of research with many potential beneficiaries.

The generation of large numbers of fast ions in ultrahighintensity laser-matter interactions, e.g., $[9,10]$, in particular promises to find wide-ranging applications including ion injectors for heavy ion accelerators [11], fast-ion-based fast ignitor schemes [12-14], and proton radiography [15]. In addition, the authors have previously reported using laseraccelerated beams of protons with a mean energy of $2 \mathrm{MeV}$ and peak proton energy of $37 \mathrm{MeV}$ to induce $(p, n)$ and $(p, \alpha)$ nuclear reactions $[16,17]$. This resulted in the production of the short-lived positron-emitting isotopes ${ }^{11} \mathrm{C}$ and ${ }^{13} \mathrm{~N}$, which are used in positron emission tomography, a noninvasive medical imaging technique. Laser-produced protons may also find applications in proton oncology for the treatment of tumors of the eye.

\footnotetext{
*Author to whom correspondence should be addressed. FAX: +441413305881.

Electronic address: K.Ledingham@physics.gla.ac.uk
}

Much of the "proof-of-principle" experimentation has involved large-scale, "single-shot," long-pulse ( $\sim 1$ ps) lasers. However, due to inherently large costs and low repetition rates it is unlikely that such laser systems will provide the quantity and range of data needed to fully elucidate the processes involved. The extension of these studies to smaller, but higher-repetition-rate lasers, maintaining focused intensities above $10^{18} \mathrm{~W} \mathrm{~cm}^{-2}$ is required to realize the development and application of the techniques described above. Laser systems providing multiterawatt pulses at a repetition rate of a few hertz have been developed at a number of laboratories worldwide. These lasers facilitate the systematic experimentation required to optimize the conditions of ion acceleration and provide a fuller understanding of the physics involved. This is required for the design of dedicated laser systems for the applications outlined.

Studies on proton acceleration by Saleh et al. at the University of Michigan have involved a laser system with a repetition rate of 1 shot per $10 \mathrm{~min}$ [18] providing 1-J, 400-fs pulses focused to an intensity of $3 \times 10^{18} \mathrm{~W} \mathrm{~cm}^{-2}$ on thinfoil targets. Fast protons with energy up to $1.5 \mathrm{MeV}$ were generated and attributed to protons from the front surface [19]. In another experiment, the same group accelerated deuterons at $6 \times 10^{18} \mathrm{~W} \mathrm{~cm}^{-2}$, and showed by inducing $(d, n)$ reactions in boron that the deuterons originated from the front target surface [20].

Recent results from Mackinnon et al. [21] using a 100-fs laser pulse and intensity greater than $10^{20} \mathrm{~W} \mathrm{~cm}^{-2}$ incident on aluminum targets showed that proton energies as high as $24 \mathrm{MeV}$ were obtained from 3- $\mu \mathrm{m}$ targets, and this energy decreased to $6.5 \mathrm{MeV}$ when $100-\mu \mathrm{m}$ targets were used. Higher proton energies in thin targets were attributed to recirculation of electrons in these targets, i.e., electrons being reflected at each of the target-vacuum boundaries and making multiple crossings of the target. In experiments at the LOA facility in France [22], $10-\mathrm{MeV}$ protons have been measured in two distinct beams, one along the laser direction and the other directed off the target normal. 


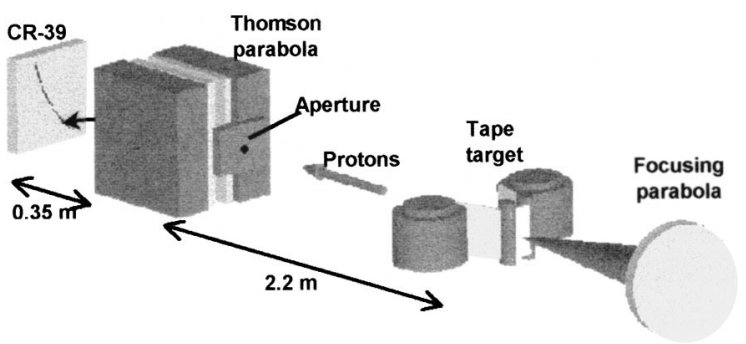

FIG. 1. Experimental arrangement for proton emission measurements.

Developments in tabletop laser technology seek to produce shorter pulse durations for a given pulse energy, thereby increasing the intensity. Due to the shorter heating time the interaction of short pulses of tens of femtoseconds with thinfoil targets could be expected to significantly change the interaction process and hence the physics of the ion acceleration. To fully understand what influence this generation of short-pulse lasers will have on the acceleration of ions and to test their feasibility for applications, it is important for experiments to be carried out in this unexplored regime.

This paper reports on measurements of proton production using pulse durations of tens of femtoseconds (60 fs) and energies below $200 \mathrm{~mJ}$. Optimum conditions for proton acceleration in terms of target material and thickness have been characterized.

\section{EXPERIMENT}

The experiment used the high-power beamline of the ASTRA Ti:sapphire laser facility [23-26] at the Rutherford Appleton Laboratory, U.K., operated in the CPA [1,2] mode. The system delivered pulses on target at normal incidence at a rate of $2 \mathrm{~Hz}$ with a wavelength centered at $790 \mathrm{~nm}$ within a target chamber held at a modest vacuum of around $10^{-5}$ Torr. The beam was focused onto the target using an F3 off-axis parabolic mirror. A full description of the characterization of the various laser parameters is the subject of another publication [27]. Briefly, the system delivered pulses on target of $200 \mathrm{~mJ}$ and duration $60 \mathrm{fs}$. The contrast ratio was measured to be $1: 10^{-6}$ or better at 10 ps before the main pulse, rising to $1: 10^{-5}$ at 4 ps and $10^{-4}$ at $1.5 \mathrm{ps}$ [27]. The maximum intensity on target was calculated to be 8.2 $\times 10^{18} \mathrm{~W} \mathrm{~cm}^{-2}$, averaging at $7 \times 10^{18} \mathrm{~W} \mathrm{~cm}^{-2}$, and corroborated by measurements of multiple ionization stages of argon [27].

In order to make use of the $2-\mathrm{Hz}$ repetition rate of the system to integrate measurements over a number of shots, two target designs were employed, a "tape drive" and a "wheel" setup, described in [27]. With both systems, any change in target position in the focal plane due to movement of the target was corrected using a retroreflecting imaging system [27].

Measurements of ion emission were made using a Thomson parabola spectrometer [28-30] backed with CR39. The experimental setup is shown in Fig. 1. CR39 is a plastic nuclear track detector which is sensitive to ions of energy greater than $100 \mathrm{keV} /$ nucleon, and insensitive to $\mathrm{x}$ rays and

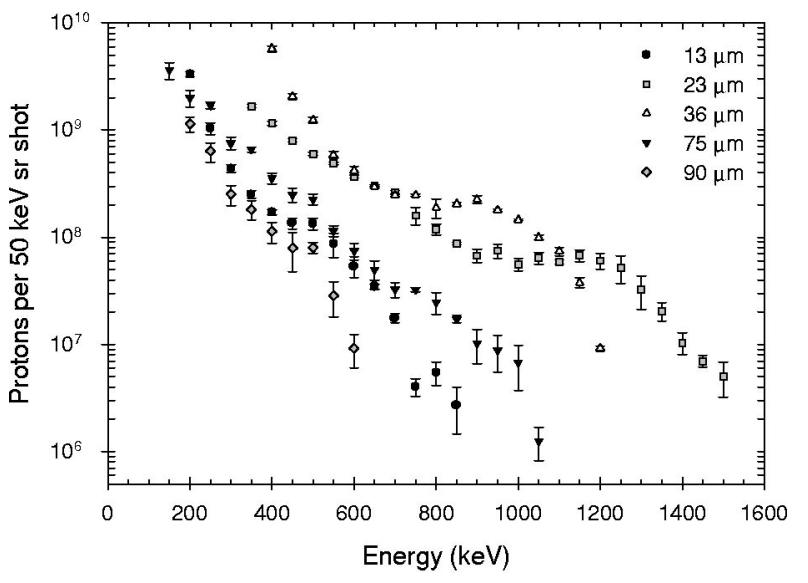

FIG. 2. Proton energy spectra emitted from Mylar targets with thickness in the range 13-90 $\mu \mathrm{m}$.

electrons. Each ion incident on the CR39 is recorded as a single pit, and since their energy can be calculated by the extent they are deflected in the spectrometer, spectral information, i.e., numbers and energy, can be obtained. Data were accumulated over 24 or 50 shots for each target.

Another experimental arrangement involved positioning CR39 detectors $\sim 4 \mathrm{~cm}$ behind the target in order to make spatially integrated measurements of proton emission, to look for any "ring" or "disk" structure $[9,31]$ in the proton beam(s) generated.

\section{PROTON MEASUREMENTS}

\section{A. Mylar target measurements}

Measurements were made of the energy spectra of ions emitted behind Mylar targets ranging in thickness from 6 to $125 \mu \mathrm{m}$. Although no signal was observed on the detector behind the Thomson parabola for the 6- $\mu \mathrm{m}$ target, a measurement of the maximum energy of the protons emitted from this target was made by covering a piece of CR39 in Mylar filters of various thicknesses such that each layer stopped protons of a certain energy. The CR39 was placed $340 \mathrm{~mm}$ behind the target along the target normal. The maximum proton energy for the 6- $\mu \mathrm{m}$ target was found to be 100 $\mathrm{keV}$, the detection threshold of CR39. No protons were detected for Mylar targets thicker than $90 \mu \mathrm{m}$.

In agreement with previous observations [32,33], the only ions detected at the rear of the target were protons. Figure 2 shows the proton spectra obtained for Mylar targets of thickness $13,23,36,75$, and $90 \mu \mathrm{m}$. In each spectrum, the highest-energy point is the cutoff energy, i.e., there are no protons above this energy. It can be seen that the maximum proton energy $1.5 \mathrm{MeV}$ was obtained for the $23-\mu \mathrm{m}$ target. It is instructive to plot the cutoff proton energies obtained as a function of target thickness, and this is shown in Fig. 3(a). From this plot, it can be seen that the optimum target thickness for maximizing the proton energy lies in the region around $20-40 \mu \mathrm{m}$. The relevance of this is discussed in Sec. V.

Spatially integrated measurements were made for a number of Mylar targets by positioning CR39 detectors $4 \mathrm{~cm}$ 

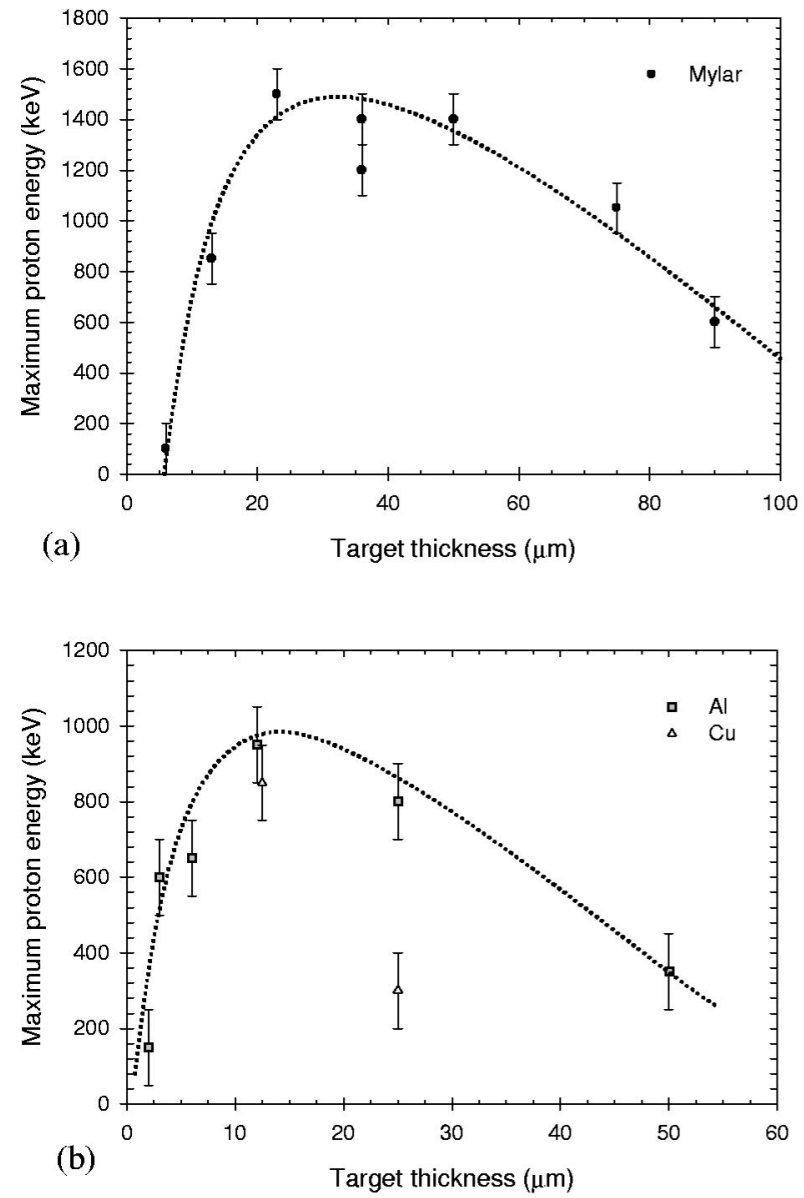

FIG. 3. Maximum proton energy obtained as a function of target thickness for (a) Mylar, (b) aluminum and copper. Dashed curves are drawn through the experimental points to guide the eye.

behind the target. The protons were found to be emitted in a cone of half angle $22^{\circ}$. The "ring" or "disk" structure observed in [9,31] was not evident in the present 200-mJ 60-fs experiments.

\section{B. Metal target measurements}

The proton measurement procedure was also carried out for metal targets, specifically, aluminum of thickness $0.8,2$, $3,6,12,25$, and $50 \mu \mathrm{m}$ and copper of thickness $0.2,0.4$, $12.5,25$, and $40 \mu \mathrm{m}$.

In the case of aluminum, no Thomson Parabola data were obtained for the 0.8 - or $2-\mu \mathrm{m}$ targets; however, measurements of maximum energy were made using the Mylar filter technique. Proton spectra for the 3-, 6-, 12-, 25-, and 50- $\mu \mathrm{m}$ targets are shown in Fig. 4. It can be seen that the maximum proton energy, $950 \mathrm{keV}$, was obtained for the $12-\mu \mathrm{m}$ target. Plotting the cutoff proton energy as a function of target thickness [Fig. 3(b)] demonstrates that the optimum thickness for $\mathrm{Al}$ is a factor of $\sim 3$ smaller than the optimum Mylar thickness.

Proton energy spectra obtained for 12.5 - and $25-\mu \mathrm{m}$-thick copper targets (Fig. 5). No protons were detected for targets of thickness 0.2 and $40 \mu \mathrm{m}$ and the highest proton energy was $850 \mathrm{keV}$ for the $12.5-\mu \mathrm{m}$ target.

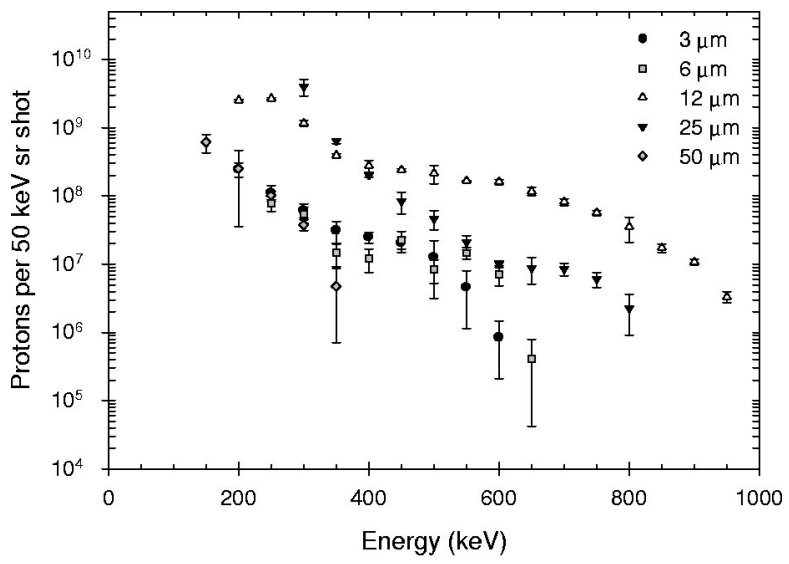

FIG. 4. Proton energy spectra emitted from aluminum targets with thickness in the range $3-50 \mu \mathrm{m}$.

Although the optimum target thicknesses appear to differ greatly for plastic (Mylar) and metal ( $\mathrm{Al}, \mathrm{Cu})$ targets, this may be explained in terms of areal densities. Figure 6 shows a plot of maximum proton energy as a function of target thickness for aluminum and Mylar, with the $x$ axis normalized to the areal density of aluminum (areal densities $\sim 1$ for Mylar, 2.7 for aluminum). Copper was not included in this plot since there were too few data points. It is clear that the optimum normalized target thicknesses for Mylar and aluminum are in good agreement, and, if this is also true in the case of copper (areal density 8.96), then the optimum target thickness will be at $\sim 4 \mu \mathrm{m}$. Targets of this thickness were not available in this experiment.

The number of protons of energy $\geqslant 400 \mathrm{keV}$ could be determined and are plotted as a function of target thickness for the Mylar, aluminum, and copper targets in Fig. 7. The maximum proton flux for Mylar is an order of magnitude greater than that of the metals. This is consistent with experiments performed on the Petawatt at LLNL [10] and suggests that, in the case of Mylar, protons are being generated from bulk hydrogen in the material and also from hydrocarbon and water impurity layers on the target surfaces, rather than from impurity layers alone.

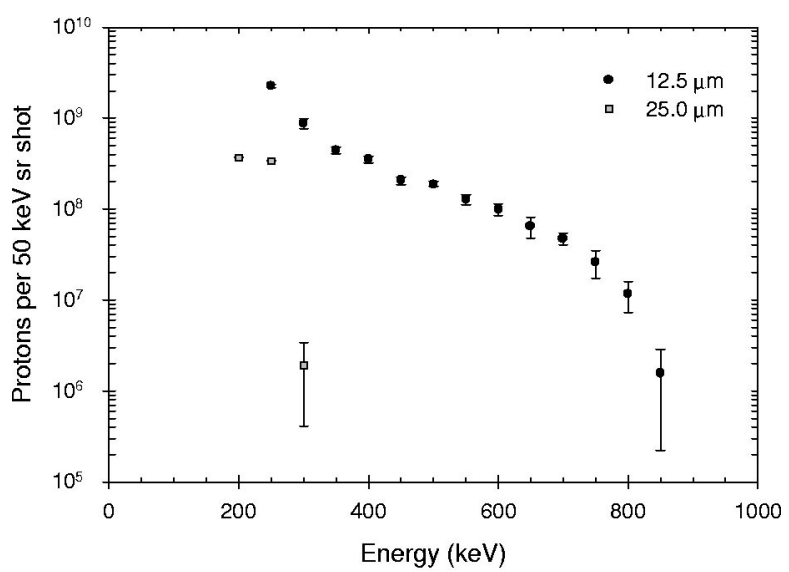

FIG. 5. Proton energy spectra obtained from copper targets of thickness 12.5 and $25 \mu \mathrm{m}$. 


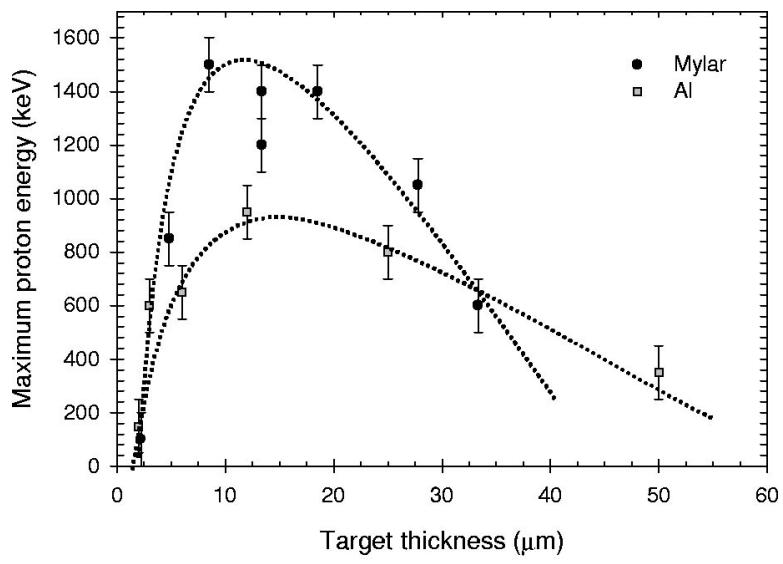

FIG. 6. Maximum proton energy as a function of target thickness for Mylar and aluminum, with the $x$ axis normalized to the areal density of aluminum. Dashed curves are drawn through the experimental points to guide the eye. Copper was not included since there were too few data points.

\section{DISCUSSION OF RESULTS}

The results presented for both plastic and metal targets show almost no proton emission from targets of thickness similar to the laser wavelength. The maximum energy increases up to an optimum thickness, and then falls off for increasing thickness. It is believed that the acceleration mechanisms for targets of thickness similar to the laser wavelength are very different from those of thicker targets $[21,32]$. The decrease in maximum proton energy after the optimum can be explained by the fast electrons (which are responsible for proton acceleration) losing energy through collisions as they travel through the target.

Mackinnon et al. [21] observed the highest proton energies from thin targets of the order of the laser wavelength. This was attributed to increased recycling of electrons in the target (reflections between front and rear surfaces) for these

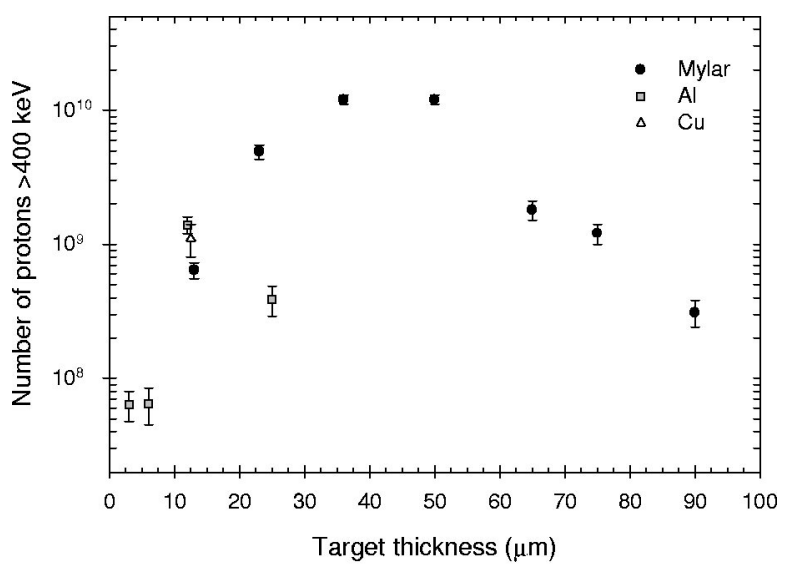

FIG. 7. Number of protons with energy $\geqslant 400 \mathrm{keV}$ as a function of target thickness for Mylar, aluminum, and copper. The flux from Mylar is an order of magnitude greater than that of the metals. targets, and was verified by particle-in-cell simulations. A sharp decrease for thicker targets up to $\sim 10 \mu \mathrm{m}$ and then a slower decrease for targets up to $100 \mu \mathrm{m}$ were also observed.

The observed differences between the two experimental observations can be explained in terms of prepulse activity. The contrast ratio of the laser used by Mackinnon et al. was of the order of $10^{10}$, which would give a maximum prepulse intensity of $10^{10} \mathrm{~W} \mathrm{~cm}^{-2}$, which would not ionize the target surface significantly prior to the arrival of the main pulse. In the present experiment, the contrast ratio was $10^{6}$ at $10 \mathrm{ps}$ before the main pulse and increased after this. A corresponding prepulse intensity of $7 \times 10^{12} \mathrm{~W} \mathrm{~cm}^{-2}$ would certainly ionize the front target surface, generating a plasma in front of the target prior to the arrival of the main pulse. Preplasma formation on the front surface effectively increases the target thickness and leads to less than perfect electron reflection. For targets of the order of the laser wavelength, preplasma formation on the rear would also be expected, which has been shown to perturb proton emission from the rear $[34,35]$. It is possible that a higher contrast ratio in the present experiment would have produced higher-energy protons for the thinnest targets used, and this will be investigated in future experiments. Mackinnon et al. stated that the proton signal was sometimes lost for thin targets, and this was attributed to an increase in prepulse activity since the prepulse proved difficult to control.

By comparing the results found here with those of Maksimchuk et al. [19], several similarities are observed. First, the proton energies obtained are very similar, including a maximum energy of about $1.5 \mathrm{MeV}$. The spectral shapes show a striking resemblance, with a flattening off of the spectrum prior to a sharp cutoff in both cases. Interestingly, even though the results obtained are extremely similar, the difference in laser parameters between the present case and that of Maksimchuk et al. is as follows: the pulse length is a factor of 7 shorter, the pulse energy a factor of 5 lower, and the intensity here a factor of 2 higher. When comparing aluminum targets, the maximum proton energy obtained here was $800 \mathrm{keV}$ compared to $1.5 \mathrm{MeV}$ in [19]. It follows that the reduced pulse length affects the acceleration mechanisms and leads to lower proton energies for similar intensities. The much lower pulse energy also affects the acceleration mechanisms, although it is believed that this should only affect the electron and subsequently proton flux. In the present case, the conversion efficiency from the laser to protons has been estimated to be $0.7 \%$. This is encouraging for the future of tabletop-laser-based proton acceleration, and further details of the implications of this finding are discussed elsewhere [27].

Maksimchuk et al. also observed a dependence of prepulse intensity on the proton energies observed, and found a maximum. The optimum prepulse intensity was $10^{15} \mathrm{~W} \mathrm{~cm}^{-2}$ at $50 \mathrm{ps}$ before the main pulse. However, Mackinnon et al. and Fritzler [36] found optimum conditions by minimizing the prepulse intensity. The laser prepulse activity obviously plays a vital role in fast-proton production, and will be investigated further in future experiments. 


\section{CONCLUSIONS}

A study of proton acceleration has been made using a tabletop 2-Hz laser system delivering 60-fs, 200-mJ pulses focused to an intensity of $7 \times 10^{18} \mathrm{~W} \mathrm{~cm}^{-2}$ on a variety of solid targets. Proton energy distributions have been obtained for various target materials and thicknesses, and the optimum conditions were obtained for Mylar targets. The optimum Mylar target thickness was found to be three times as thick as that of $\mathrm{Al}$ targets. The lack of protons for very thin targets can be explained in terms of preplasma formation due to the $10^{6}$ contrast ratio of the laser system. By comparing the results herein to other recent experiments, it can be seen that the laser pulse duration plays an important role in proton production. The angular distribution of proton emission was very different compared to that in picosecond experiments.
In the present experiment, no ring or disk structure was observed. These results are promising for tabletop highrepetition-rate laser proton acceleration in terms of conversion efficiency to protons.

Experimental investigations on laser-induced proton acceleration are greatly enhanced by the use of high-repetitionrate tabletop lasers. This is reflected in the present study, in which a large quantity of systematic and reproducible measurements were made to resolve the optimum target conditions for proton acceleration.

The experimental data from large-scale single-shot systems largely complements that from high-repetition tabletop instruments. The single-shot systems will invariably provide the highest-intensity conditions and hence "proof of principle" experimentation. On the other hand, the highrepetition-rate lasers facilitate use of the systematic and comprehensive conditions for fully understanding the physics involved.
[1] D. Strickland and G. Mourou, Opt. Commun. 56, 219 (1985).

[2] M. D. Perry and G. Mourou, Science 264, 917 (1994).

[3] I. N. Ross et al., Laser Part. Beams 17, 331 (1999).

[4] K. W. D. Ledingham et al., Phys. Rev. Lett. 84, 899 (2000).

[5] T. E. Cowan et al., Phys. Rev. Lett. 84, 903 (2000).

[6] T. W. Phillips et al., Rev. Sci. Instrum. 70, 1213 (1999).

[7] K. W. D. Ledingham and P. A. Norreys, Contemp. Phys. 40, 367 (1999).

[8] M. Tatarakis et al., Nature (London) 415, 280 (2002).

[9] E. L. Clark et al., Phys. Rev. Lett. 84, 670 (2000).

[10] R. A. Snavely et al., Phys. Rev. Lett. 85, 2945 (2000).

[11] K. Krushelnick et al., IEEE Trans. Plasma Sci. 28, 1184 (2000)

[12] M. Tabak et al., Phys. Plasmas 1, 1626 (1994).

[13] M. Roth et al., Phys. Rev. Lett. 86, 436 (2001).

[14] R. Kodama et al., Nature (London) 412, 798 (2001).

[15] M. Borghesi et al., Plasma Phys. Controlled Fusion 43, A267 (2001).

[16] M. I. K. Santala et al., Appl. Phys. Lett. 78, 19 (2001).

[17] I. Spencer et al., Nucl. Instrum. Methods Phys. Res. B 183, 449 (2001).

[18] N. Saleh et al., Rev. Sci. Instrum. 71, 2305 (2000).

[19] A. Maksimchuk et al., Phys. Rev. Lett. 84, 4108 (2000).

[20] K. Nemoto et al., Appl. Phys. Lett. 78, 595 (2001).
[21] A. J. Mackinnon et al., Phys. Rev. Lett. 88, 215006 (2002).

[22] V. Malka (private communication).

[23] C. J. Hooker and A. Marshall, Central Laser Facility, Rutherford Appleton Laboratory Annual Report 1998-99, 1999, p. 190.

[24] A. J. Langley et al., Central Laser Facility, Rutherford Appleton Laboratory Annual Report 1998-99, 1999, p. 186.

[25] A. J. Langley et al., Central Laser Facility, Rutherford Appleton Laboratory Annual Report 1998-99, 1999, p. 187.

[26] A. J. Langley et al., Rutherford Appleton Laboratory Central Laser Facility Annual Report 1999/2000, 2000, p. 196.

[27] P. McKenna et al., Rev. Sci. Instrum. 73, 4176 (2002).

[28] M. R. Wehr, J. A. Richards, Jr., and T. W. Adair III, Physics of the Atom (Addison-Wesley, Reading, MA, 1983).

[29] K. Krushelnick et al., Phys. Rev. Lett. 83, 737 (1999).

[30] E. L. Clark et al., Phys. Rev. Lett. 85, 1654 (2000).

[31] Y. Murakami et al., Phys. Plasmas 8, 4138 (2001).

[32] J. T. Mendonça, J. R. Davies, and M. Eloy, Meas. Sci. Technol. 12, 1801 (2001)

[33] J. Badziak et al., Phys. Rev. Lett. 87, 215001 (2001).

[34] A. J. Mackinnon et al., Phys. Rev. Lett. 86, 1769 (2001).

[35] M. Roth et al., Nucl. Instrum. Methods Phys. Res. A 464, 201 (2001).

[36] S. Fritzler (private communication). 